\title{
Which Disposable Chest Electrode?
}

\author{
P. J. B. HUBNER,* M.B., M.R.C.P.
}

British Medical fournal, 1969, 3, 507-510

Summary: Chest electrodes are preferred to limb $\checkmark$ electrodes for cardiac monitoring, as limb movements are not restricted and produce less interference of the E.C.G. trace. Eight types of disposable chest electrodes were investigated to compare their performance, skin reactions, cost, ease of application, size, and skin-electrode impedance.

Elema-Schonander electrodes were found to be the most efficient and the most expensive. In their application care was required to avoid severe skin reactions.

Dracard electrodes were simple to attach, worked well without severe skin reactions, and were cheap. They are recommended for routine use. Smith and Nephew electrodes, a type of "multipoint electrodes" which do not require electrode jelly, frequently produced severe skin reactions, making them unsuitable for monitoring for periods exceeding 12 hours.

\section{Introduction}

Cardiac monitoring with E.C.G. oscilloscopes is often used routinely in the management of acute myocardial infarction, after cardiac surgery, after resuscitation from cardiac arrest, in the treatment of arrhythmias, and for the assessment of cardiac pacing. The skin electrodes used may be positioned on the limbs or on the chest. Chest electrodes are preferred because movements of the limbs are not restricted and produce less interference of the E.C.G. trace. With monitor alarm systems false-alarms produced by interference from limb movements are less frequent with electrodes positioned on the chest than on the limbs. Chest electrodes may be disposable or nondisposable. The former allow a fresh electrode to be used for each patient, and cleaning of the electrodes after use is not necessary.

The desirable features of disposable chest electrodes, henceforth termed simply electrodes, are:

(1) Immediately after attachment to the skin they should give a satisfactory E.C.G. trace-that is, a thin one with a steady base-

- Junior M.R.C. Research Fellow and Honorary Medical Registrar, Medical Research Council's Cardiovascular Research Unit, and De-
partment of Medicine, Royal Postgraduate Medical School, Hammerpartment of Medicine, Royal Po
smith Hospital, London W.12. line free from alternating current (A.C.) interference. After movements by the patient there should be minimal or no interference with the trace.

(2) They should produce a satisfactory trace for up to a week without need for electrode replacement.

(3) They should produce no skin reaction.

(4) They should be cheap.

(5) They should be easy to apply, for they will sometimes be applied during or after an emergency, frequently by non-medical staff.

(6) They should have a simple connexion between the electrode and the lead from the monitor.

(7) They should have a small external surface area and radiological opacity so as not to obscure examination of the heart, recording of a standard E.C.G., or examination of chest $x$-ray films.

(8) They should have a low skin-electrode impedance, as on theoretical grounds features 1 and 2 are then more likely to be satisfied.

The duration of a week for electrode attachment is arbitrary, but after acute myocardial infarction patients are often monitored for this period. Eight types of electrodes were investigated to assess these features.

\section{Materials and Methods}

All of the eight types of electrodes (Table I) were considered disposable, though the electrode discs of DE and TEM may be used again. K-Y jelly (Johnson and Johnson)-a nonirritant and efficient jelly (Lewes, 1965a)-was used for electrodes requiring electrode jelly. All tests were performed on the same test monitor, a Cardiac Recorders Cardiorater 64, which has an input impedance of 5 megohms. The study was made in a coronary care unit and adjacent medical wards. Sources of A.C. interference-for example, monitor and other mains cables-were kept well away from the beds.

Each type of electrode was assessed on 10 patients, two of whom were women (except for SN electrodes when there was only one woman). All patients were having bed-rest and sat out only to use the commode. No patient received external cardiac massage, D.C. countershock, or cardiac pacing during the study.

TABLE I.-Electrodes Investigated

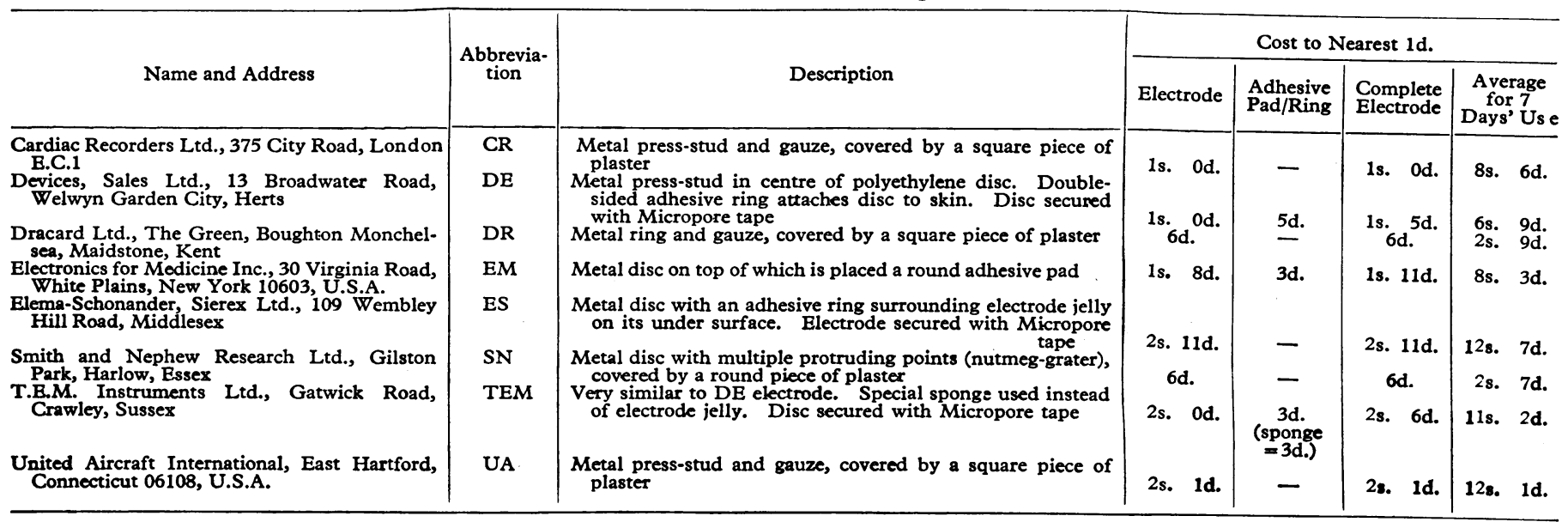




\section{Assessment of Initial Trace}

When necessary the skin of the proposed electrode sites was shaved. It was then rubbed with a piece of gauze soaked with ether, until mild erythema appeared, to lower the skin electrical resistance (Boter et al., 1966). Four electrodes were attached to the skin; the time taken, including application of electrode jelly, was recorded on a stop-watch. Two electrodes were placed on each side of the sternum, except for DE electrodes, which were positioned two down the sternum and one under each nipple, as recommended by Fluck and Burgess (1966), who described these electrodes.

The monitor leads-right arm, right leg, left arm, and left leg (RA, RL, LA, and LL)-were connected to electrodes at the appropriate corner of a rectangle formed by the four electrodes. With DE electrodes, LA and RA passed to the electrodes at the upper and lower ends of the sternum and RL and LL to the electrodes beneath the nipples of the appropriate side. The leads were always connected in this way, so that the electrodes responsible for the E.C.G. trace on a given channel of the monitor were known.

The trace on the monitor screen was examined on channels I, II, III, aVR, aVL, and aVF with the gain control left on maximum. If the trace was unsatisfactory-that is, the baseline on any of the six channels instead of being a thin line was broad and appeared "fuzzy" due to $50 \mathrm{~Hz}$ A.C. interference -an "earthing wire" was used. A wire with a bulldog-clip at each end was connected from a metal contact on the bed to one on the monitor. If A.C. interference persisted it was concluded to be due to faulty electrode(s). As the electrodes responsible for the trace on the channels observed to have A.C. interference were known, the electrode(s) at fault and needing to be replaced could be identified. After each electrode replacement the skin was again cleaned and the trace on all the channels inspected. The need for use of the " earthing wire," or electrode replacement(s), or both, to obtain a satisfactory trace was recorded.

Skin-electrode impedance was next measured for each of the four electrode pairs-LA-RA, RA-RL, RL-LL, and LL-LAby a method (Fig. 1) suggested by H. S. Wolff (personal communication). A simple method of employing the patient's own E.C.G. signal, it measured impedance differences of $500 \mathrm{ohms}$. The impedance measurement was the average of two readings, ascending and descending the resistance scale.

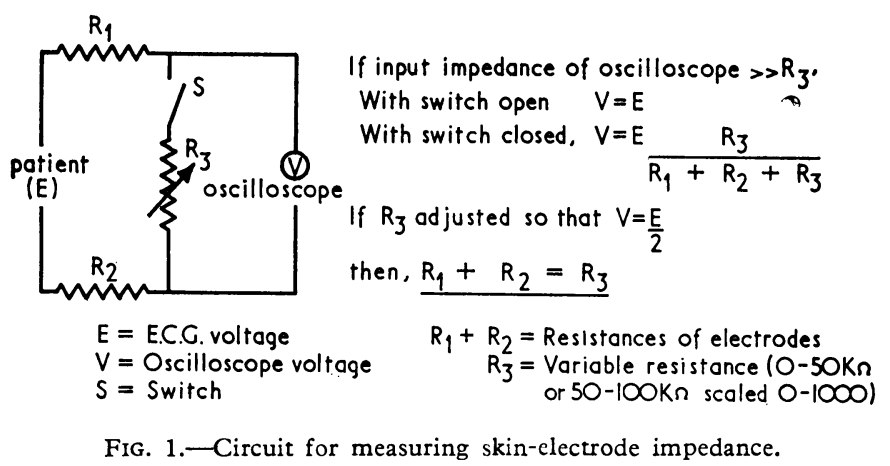

\section{Securing Electrodes and Monitor Leads}

DE and TEM electrodes were secured with four strips of $\frac{1}{2}$-in. $(1 \cdot 3-\mathrm{cm}$.) Micropore tape (3M Company Ltd.) placed over the periphery of the disc and the adjacent skin. Three strips of 1 -in. $(2 \cdot 5-\mathrm{cm}$.) Micropore tape were placed over each ES electrode; after the first two patients a further strip of 1-in. $(2 \cdot 5-\mathrm{cm}$.) tape was also placed under each ES electrodeintermediate lead connexion. The time taken to secure the electrodes was recorded on a stop-watch. The other electrodes did not require securing with Micropore.
To prevent any strain on the leads being transmitted to the electrodes the monitor leads were collected in a loop and secured with zinc oxide plaster in the epigastrium. The monitor cable was then removed from the test monitor and inserted into the bedside monitor.

\section{Subsequent Trace}

On each of the next seven days the electrodes were assessed. The monitor cable was inserted into the test monitor, and with the gain control on maximum the trace on channels I-aVF was inspected. If the trace was unsatisfactory the measures previously described were performed. The day and need for use of " earthing wire" or electrode replacement, or both, were recorded. The monitor leads were resecured if they became loose.

Though the patient was visited only once daily specifically to assess the electrodes, if one became displaced at another time it was replaced. The replacement was recorded on the next day's electrode observations.

\section{Skin Reactions}

After a satisfactory trace had been seen on the seventh day the electrodes were removed and the skin was inspected for reactions, which were classified into: grade 0 , no reaction; grade 1, erythema and/or up to two acneiform spots ; grade 2, reaction intermediate between grades 1 and 3 , reaction with three or more acneiform spots; and grade 3 , severe reaction with skin ulceration or blistering, requiring a skin dressing.

\section{Results}

Electrode Performance (Table II and Fig. 2).-The initial trace was most often unsatisfactory with DE and TEM electrodes, which required the most frequent use of the " earthing wire" and electrode replacement. The duration of a satisfactory trace was clearly the longest with ES electrodes, which required only very infrequent electrode replacements and use of the "earthing wire." The largest number of electrode replacements was for CR electrodes. The " earthing wire" was needed in over half of the electrode tests on CR, DE, TEM, and UA electrodes. Seven of the $11 \mathrm{SN}$ electrode replacements were for fracture at the electrode of the wire passing from it to the electrode-lead connexion. The wire of one EM electrode also fractured.

TABLE II.-E.C.G. Trace

\begin{tabular}{c|c|c|c|c}
\hline \multirow{2}{*}{ Type } & \multicolumn{2}{|c|}{ Initial Trace } & \multicolumn{2}{c}{ Subsequent Trace } \\
\cline { 2 - 5 } & $\begin{array}{c}\text { Earthing Wire } \\
\text { Required }\end{array}$ & $\begin{array}{c}\text { Electrodes } \\
\text { Replaced }\end{array}$ & $\begin{array}{c}\text { Earthing Wire } \\
\text { Required }\end{array}$ & $\begin{array}{c}\text { Electrodes } \\
\text { Replaced* }\end{array}$ \\
\hline CR & 1 & 0 & 36 & 45 \\
DE & 3 & 4 & 37 & 22 \\
DR & 1 & 0 & 21 & 15 \\
EM & 1 & 0 & 22 & 16 \\
ES & 0 & 1 & 2 & 2 \\
SN & 1 & 0 & 20 & 11 \\
TEM & 3 & 9 & 41 & 19 \\
UA & 1 & 0 & 37 & 18
\end{tabular}

Number of times that the arthing wire was needed, and total number of electrodes which were replaced to obtain a satisfactory E.C.G. trace initially, and subsequently over one week with 10 patients.

10 excluded.

Skin Reactions (Table III).--Sixty per cent. of SN electrodes produced a severe (grade 3 ) reaction of ulceration and sometimes pustular formation of the skin in contact with the electrode plate. Three out of eight ES electrodes on the first two patients produced severe erythema with a blister of the skin under the connexion of the electrode to the intermediate lead. There was a green discoloration of this part of the 
electrode and ensheathing cover of the intermediate lead, perhaps caused by corrosion of the metal by sweat. Further reactions of this type were subsequently prevented by placing a strip of Micropore tape underneath the electrode-intermediate lead connexion. CR, DR, EM, and UA electrodes did not produce a severe reaction. UA electrodes gave the least skin reactions of all the electrodes tested. Acneiform spots were the common type of skin reaction with DR electrodes.

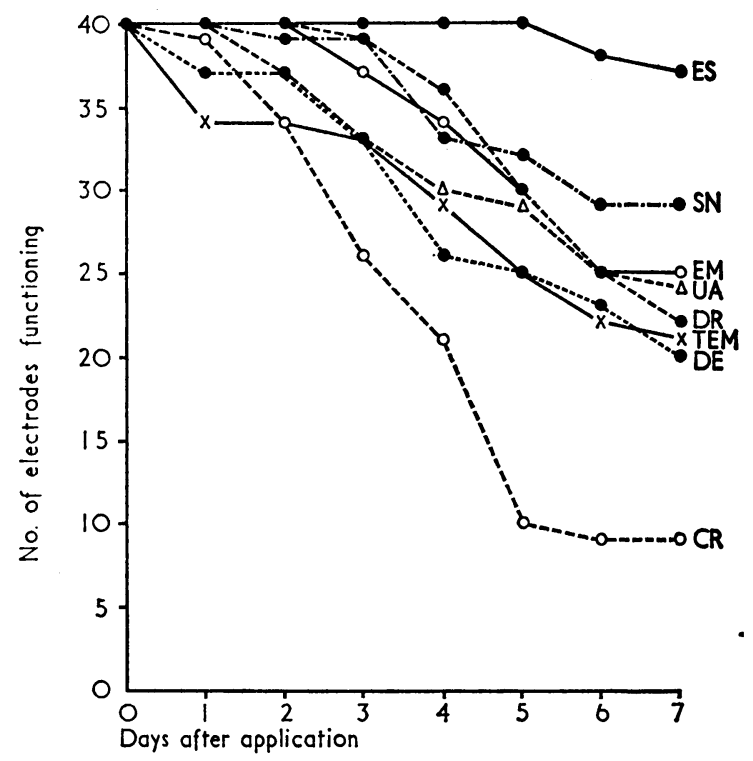

Fig. 2.-Electrode duration. Course of 40 electrodes achieving a satisfactory initial trace on 10 patients, to show the number still functioning-that is, not requiring to be replaced-one to seven days later.

\begin{tabular}{c|c|c|c|c}
\multicolumn{5}{c}{ TABLE III.-Skin Reactions } \\
\hline \multirow{2}{*}{ Type } & \multicolumn{4}{|c}{ Skin Reaction } \\
\cline { 2 - 4 } & Grade 0 & Grade 1 & Grade 2 & Grade 3 \\
\hline CR & 14 & 18 & 8 & 0 \\
DE & 18 & 14 & 5 & 3 \\
DR & 20 & 16 & 4 & 0 \\
EM & 22 & 17 & 1 & 3 \\
ES & 25 & 12 & 0 & 24 \\
SN & 1 & 8 & 7 & 1 \\
TEM & 12 & 25 & 2 & 0
\end{tabular}

Skin reactions at 40 electrode sites on 10 patients after 1 week of monitoring.

Cost.-Table I shows the price per complete electrode and the average cost per patient for one week's monitoring, allowing for electrode replacements of Table II. The cost of Micropore tape and electrode jelly was not included in this assessment. With DE, EM, and TEM electrode replacements the original electrode with only a fresh adhesive pad/ring was used. A new sponge was used with each TEM electrode replacement except those necessary on the day of initial application. There was a wide range in cost of an electrode, from $6 d$. for DR and $S N$ electrodes to $2 \mathrm{~s}$. 11d. for an ES electrode. A week's monitoring was cheapest when using SN and DR electrodes (2s. 7d. and 2s. 9d.) and dearest with ES and UA electrodes (12s. 7d. and 12s. 1d.).

Ease of Electrode Application and Connexion to Monitor Lead.-The ease of application was assessed by the time required to apply four electrodes to the skin and by the number of "steps" involved in this process (Table IV). For example, with CR, DR, and UA electrodes the removal of the plaster cover, the application of electrode jelly, and attachment of the electrode to the chest involved three "steps." The application of DE and TEM electrodes was more complicated; it involved more "steps" and consequently took longer. ES and SN electrodes were the simplest and quickest to attach, neither requiring electrode jelly to be applied. The test monitor's leads had cylindrical terminals of 3-mm. diameter for which DR and SN electrodes had a direct connexion. All other electrodes required an intermediate clip or lead between the electrode and monitor lead terminal. Only a bare-ended wire emerged from EM electrodes, to which a connexion had to be applied before their use.

TABLE IV.-Ease of Electrode Application

\begin{tabular}{|c|c|c|c|c|}
\hline \multirow[t]{2}{*}{ Type } & \multirow{2}{*}{$\begin{array}{l}\text { No. of Steps } \\
\text { in Electrode } \\
\text { Application }\end{array}$} & $\begin{array}{c}\text { Time to } \\
\text { Apply } 4 \\
\text { Electrodes }\end{array}$ & $\begin{array}{c}\text { Time to } \\
\text { Secure } 4 \\
\text { Electrodes }\end{array}$ & $\begin{array}{l}\text { Total Time } \\
\text { to Apply } 4 \\
\text { Electrodes }\end{array}$ \\
\hline & & \multicolumn{3}{|c|}{ Seconds } \\
\hline $\begin{array}{l}\text { CR } \\
\text { DE } \\
\text { DR } \\
\text { EM } \\
\text { ES } \\
\text { SN } \\
\text { TEM } \\
\text { UA }\end{array}$ & $\begin{array}{l}3 \\
6 \\
3 \\
4 \\
3 \\
2 \\
6 \\
3\end{array}$ & $\begin{array}{r}80 \\
105 \\
75 \\
103 \\
33 \\
59 \\
87 \\
109\end{array}$ & $\frac{\overline{87}}{\frac{30}{87}}$ & $\begin{array}{r}80 \\
192 \\
75 \\
103 \\
63 \\
59 \\
174 \\
109\end{array}$ \\
\hline
\end{tabular}

Times: the averages for four electrodes on 10 patients, to nearest second.

Electrode Size.-With an adhesive pad or Micropore tape the external surface area of all electrode types was similar, ranging from 1,444 sq. mm. for CR and UA electrodes to 1,980 sq. $\mathrm{mm}$. for ES electrodes. There was a greater variation in the size of radiological opacity: from 45 and 79 sq. $\mathrm{mm}$. for DR and $\mathrm{DE}$ electrodes to 744 sq. $\mathrm{mm}$. for ES electrodes.

Skin-electrode Impedance (Table V).-If skin-electrode impedance is low a trace is more likely to be free from A.C. interference (Hill, 1965). As the input impedance of monitoring equipment commonly used in Britain is not particularly high the monitors are sensitive to raised skin-electrode impedance. ES electrodes were clearly superior to other types, as $70 \%$ of electrode pairs had impedance values of less than 5 Kohms.

\begin{tabular}{l|c|c|c|c|c}
\multicolumn{7}{c}{ TABLE V.-Skin-electrode Impedance } \\
\cline { 2 - 6 } Type & \multicolumn{5}{|c}{ Skin-electrode Impedance (K $\Omega)$} \\
\cline { 2 - 6 } & $<5$ & $5-25$ & $25-50$ & $50-75$ & $>75$ \\
\hline CR & 6 & 16 & 8 & 2 & 8 \\
DE & 6 & 13 & 10 & 6 & 5 \\
DR & 6 & 12 & 11 & 4 & 7 \\
EM & 4 & 12 & 9 & 5 & 10 \\
ES & 28 & 8 & 2 & 2 & 0 \\
SN & 1 & 11 & 18 & 3 & 7 \\
TEM & 1 & 17 & 6 & 7 & 9 \\
UA & 4 & 10 & 14 & 5 & 7
\end{tabular}

Values"of skin-electrode impedance of 40 pairs of electrodes on 10 patients.

\section{Discussion and Conclusions}

This study has shown a wide range in performance, skin reactions, and cost of eight types of disposable chest electrodes. Different results might have been obtained with other forms of electrode jelly and monitoring equipment. The particular features of the various types of electrodes are as follows:

Elema-Schonander Electrodes.-These electrodes were the most efficient and the most expensive of those tested (Tables I and II). Their success is believed to be due to their relatively large dark-brown-coated metal electrode, which is in direct contact with the skin. As the electrodes lay flush to the skin covered with Micropore tape none became displaced. The size of their metal electrode gave them a relatively large radiological opacity. As they are supplied with electrode jelly already on them, their application was simple and quick. Their very low skin-electrode impedance was not due to their own jelly, as identical values were found when it was replaced by K-Y jelly. When a strip of Micropore tape was placed on the skin under the electrode-lead connexion severe skin reactions did not occur. 
Dracard Electrodes.-These electrodes are recommended for routine use. They are simple to apply and worked well initially and over a week. They did not produce severe skin reactions, had the smallest radiological opacity, and cost only $6 \mathrm{~d}$. each. After 48 to 72 hours their plaster began to wrinkle, allowing the electrode jelly to dry, so that the trace became unsatisfactory and some electrodes then needed replacing. The number of replacements, however, was not excessive (Table II). Skin reactions due to the edge of the electrode gauze pressing into the skin (Hill and White, 1968) were rare.

Smith and Nephew Electrodes.-SN electrodes are a type of "multipoint electrodes," which have a nutmeg-grater electrode plate whose points penetrate the epidermis, so that electrode jelly is unnecessary (Lewes, 1965b). They were the simplest and quickest electrodes to apply and produced a satisfactory initial trace of adequate duration. A pricking sensation was commented on by some patients. Severe skin reactions appearing in 12 to 48 hours were far commoner with $\mathrm{SN}$ electrodes than with any other electrode type (Table III). The reactions were partly due to the electrode plaster, for they were less common when these electrode plates were secured with Dracard plaster. Some reactions were pustular, but swabs of the lesions were sterile on culture. Though reactions have not occurred with their use over a few hours (D. W. Hill, personal communication, 1969), for periods exceeding 12 hours monitoring with SN electrodes is considered unsuitable.

Devices and TEM Electrodes.-These electrodes consist of a metal stud within the dome of a polyethylene cup which contains electrode jelly or a sponge. This design is claimed to reduce movement artifacts (Boter et al., 1966). The small size of metal electrode and its lack of direct contact with the skin, however, are believed to be responsible for a poor initial performance (Table II). Their method of attachment to the skin was relatively complicated, but they rarely became displaced, so that electrode replacements were usually for correction of A.C. interference. The positioning of DE electrodes below the nipple (Fluck and Burgess, 1966) is unsuitable for women, as the edge of the electrode disc tends to cut into the skin of the breast. Monitoring was relatively expensive with TEM electrodes, and the use of sponge instead of electrode jelly was not found to have any special value.

Cardiac Recorders Electrodes.-The very short duration of action of CR electrodes (Fig. 2) was due to the electrodes becoming displaced. The plaster adhesiveness was poor, and the direct attachment of the lead terminal above the electrode caused the electrode to rise up off the chest with movements of the lead. CR electrodes were easy to apply, gave a satisfactory initial trace, and did not produce severe skin reactions.

Electronics for Medicine and United Aircraft International. -Both these electrodes gave a satisfactory initial trace of average duration. Neither produced severe skin reactions; UA electrodes gave the least skin reactions of all the electrode types. From EM electrodes appears a bare-ended wire to which a connexion has to be attached before monitoring can start, which is inconvenient and time-wasting. EM electrodes tended to slip out from under the adhesive pad attaching them to the skin. Monitoring with UA electrodes was relatively expensive.

I would like to thank Dr. M. J. Goldberg, who suggested that a Which? report on electrodes be attempted; Professor J. P. Shillingford for allowing the study to be made with patients under his care; Dr. D. Lewes, Dr. D. W. Hill, Mr. P. A. Burgess, Mr. C. Mills, and Mr. H. S. Wolff for their technical advice; Miss J. Powell for drawing the diagrams ; Cardiac Recorders Ltd. for loan of the test monitor; and the electrode manufacturers for a generous free supply of electrodes. The form and price of the electrodes detailed in Table I are those that were current during the study.

\section{REFERENCES}

Boter, J., Den Hertog, A., and Kuiper, J. (1966). Medical and Biological

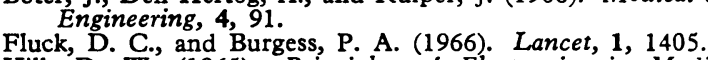

Hill, D. W. (1965). Principles of Electronics in Medical Research, p. 160. London, Butterworths.

Hill, D. W., and White, E. (1968). Medical and Biological Engineering, 6, 527.,

Lewes, D. (1965a). British Heart fournal, 27, 105.

Lewes, D. (1965b). Lancet, 2, 17.

\section{Preliminary Communications}

\section{Low Serum Pseudocholinesterase Levels Complicating Treatment with Phenelzine}

British Medical fournal, 1969, 3, 510-512

\begin{abstract}
Cummary : Four patients undergoing treatment with $\checkmark$ phenelzine developed low serum pseudocholinesterase levels. In one of the patients an apnoea of one hour followed modified electric convulsion therapy. In three cases investigations showed that the serum pseudocholinesterase levels returned to normal after withdrawal of phenelzine.
\end{abstract}

\section{INTRODUCTION}

The monoamine oxidase inhibitors form a valuable method of treating depressive illness, particularly of the neurotic type. Unfortunately, as well as exerting beneficial effects, these drugs are associated with sensitivity reactions-for example, jaundice and other potentially dangerous complications-due to drug interactions such as those which occur with pressor agents
(Dally, 1962), tricyclic antidepressants (Singh, 1960 ; Nymark and Nielsen, 1963), and analgesics (Shee, 1960 ; Pells Cocks, and Rossmore-Rowe, 1962 ; Taylor, 1962).

When phenelzine was introduced in 1959 Bleaden and Czekanska (1960) reported the cases of two patients who, while undergoing treatment with phenelzine, were given electric convulsion therapy (E.C.T.) and developed " alarming" reactions a few minutes after an apparently normal recovery from their seizure. Disordered respiration-in one case breathing stopped, and in the other it became shallow-and a slow and weak pulse were noted. Both patients responded to injections of nikethamide and positive-pressure ventilation with oxygen. Of the two cases one was taking phenelzine at the time of the reaction, while the other had discontinued treatment five days previously after a month on phenelzine $135 \mathrm{mg}$. daily, a dose substantially above that usually prescribed. As E.C.T. in one patient was modified and in the other was unmodified, the authors assumed that the reaction was not due to phenelzine altering the action of thiopentone or suxamethonium but was the result of the E.C.T. itself.

Goldman (1960), discussing this report, considered the reaction characteristic of "respiratory sluggishness" associated 